\title{
The origin of a double insertion of homogeneously staining regions in the house mouse (Mus musculus musculus)
}

\author{
S. I. Agulnik, \\ P. M. Borodin, \\ I. P. Gorlov, \\ T. Yu. Ladygina and \\ S. D. Pak
}

\author{
Institute of Cytology and Genetics, Siberian \\ Department of the Academy of Sciences of the \\ U.S.S.R., Novosibirsk 630090 U.S.S.R.
}

\begin{abstract}
A high resolution analysis of the G-band patterns of normal and aberrant chromosomes 1 , bearing two linked insertions of homogeneously staining regions (HSRs) in the house mouse (Mus musculus musculus) reveals the inverted pattern of the euchromatic region between the HSRs. On the basis of this analysis a hypothesis on the origin of the aberrant chromosome is put forward: the double insertion is a result of an inversion of the chromosome 1 of $M$ us musculus domesticus bearing a single insertion of HSR. The proximal breakpoint is localized inside the HSR, and the distal one between subbands $\mathrm{E} 3$ and $\mathrm{E} 4$.
\end{abstract}

\section{INTRODUCTION}

Several populations of the house mouse (Mus musculus) from Western Europe to the Far East have been shown to be polymorphic for chromosome 1 carring one or two insertions of homogeneously staining regions (HSR) (Agulnik et al., 1988, Traut et al., 1984, Yakimenko, Korobitsina, 1988). This double insertion is characteristic to Asian populations of M.m. musculus, while the single one is found in M.m. domesticus. An homology between the DNA sequences belonging to the HSR of M.m. domesticus and M.m. musculus has been demonstrated (Weith et al., 1987). The HSR was shown to represent amplified and probably rearranged DNA sequences (Weith et al., 1987). A coincidence in location of the single insertion of M.m. domesticus and the proximal insertion of M.m. musculus was demonstrated: both of them were localized at the distal end of subband C5 (Agulnik et al., 1988, Traut et al., 1984). The total length of insertions has a wide range of variation in both subspecies: from 6 to 30 per cent of the size of normal chromosome 1 in M.m. domesticus (Traut et al., 1984) and from 30 to 50 per cent in M.m. musculus (Agulnik et al., 1988).

The question is: do these insertions of HSR arise independently in both subspecies as a result of amplification evens, or are they originated from a common ancestor? High resolution G-banding of prometaphase chromosome 1 carrying double insertion of HSR allows a solution to this problem.

\section{MATERIALS AND METHODS}

Wild house mouse (M.m. musculus) heterozygous for a double insertion of HSR was trapped near Novosibirsk (Western Siberia, U.S.S.R). The abnormal Chromosome 1 was maintained on the genetic background of CBA/Lac strain in the heterozygous state. Mitotic cells were obtained from embryonic fibroblasts of heterozygous animals. Freshly dissolved ethidium bromide (Sigma) was introduced to the culture in a final concentration of $5 \mu \mathrm{g} / \mathrm{ml}$ for $6 \mathrm{~h}$. Cultured cells were treated with Colcemid $(0.075 \mu \mathrm{g} / \mathrm{ml})$ for $2 \mathrm{~h}$ prior to harvest. Cells were spun down and suspended in $0.075 \mathrm{M} \mathrm{KCl} 20 \mathrm{~min}$ at $37^{\circ} \mathrm{C}$ and fixed four times with $1: 3$ acetic acid/methanol. The cells were spread on clean slides by air drying without flaming, and stained for 5 mins in 5 per cent Giemsa (Merk) dissolved in phosphate buffer ( $p$ H 6.8). G-bands were obtained by mild treatment with $0 \cdot 125$ per cent trypsin for $10 \mathrm{~s}$, using chromosome slides that had been kept at room temperature for 7 days (Ikeuchi, 1984). 


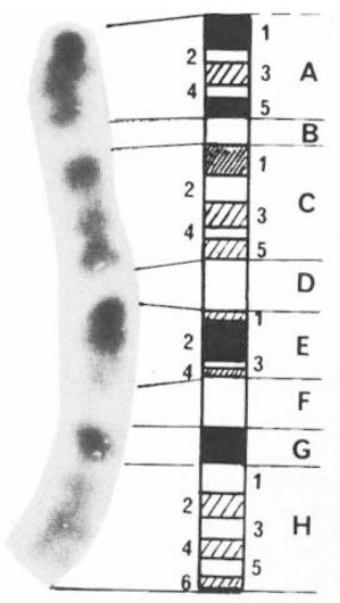

A

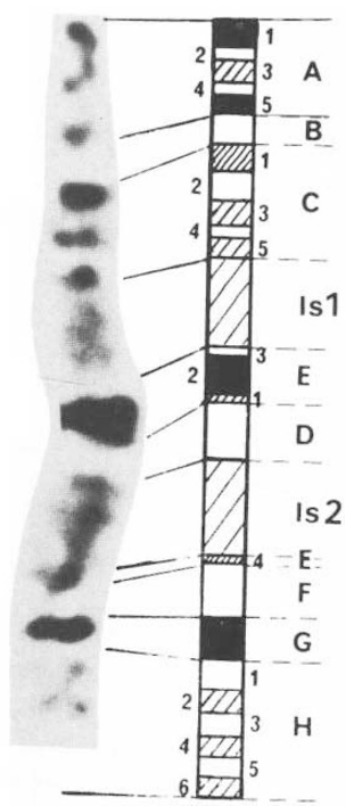

B

Figure 1 Localization of the double insertion of HSR on the cytological map of the chromosome 1 of the house mouse. A=standard chromosome 1, B = aberrant chromosome 1 .

\section{RESULTS}

Fig. 1 shows the prometaphase G-band pattern of the normal and aberrant chromosome 1 of $M . m$. musculus. The proximal insertion is located more distally than subband $\mathrm{C} 5$, confirming our earlier findings. On the basis of routine G-banding of metaphase chromosomes, the distal insertion of M.m. musculus was localized between sub-bands E3 and E4 (Agulnik et al., 1988). However, the high resolution analysis allowed us to visualize a rearranged banding pattern between the two HSR insertions. The dark sub-band E3, not the light band $\mathrm{D}$, determines the proximal insertion. The band $\mathrm{D}$ is localized more distally than $\mathrm{E}$, and adjoins the distal insertion of HSR.

Thus, the euchromatic region, demarcated by the insertions in the aberrant chromosome 1 of M.m. musculus is obviously inverted. From the point of view of these data, new symbols for these chromosomal rearrangements are proposed according to the Rules for Nomenclature of Chromosome Anomalies (Lyon, 1981): Is(HSR; 1C5) $1 \mathrm{Icg}$ - for the proximal insertion, Is(HSR; 1D) $2 \mathrm{Icg}$ - for distal one, In(1) $1 \mathrm{Icg}$ - for the inverted region including bands D, E1-E3 and insertion Is(HSR; 1D)2Icg.

\section{DISCUSSION}

The finding of the inversion in chromosome 1, carrying the two linked insertions of HSR suggests that this chromosome, which is characteristic of M.m. musculus, originates from the chromosome carrying the single insertion of HSR, which is characteristic of M.m. domesticus. Indeed, DNA from the HSR of these two subspecies was shown to be homologous, location of the single insertion of M.m. domesticus coincides with the location of the proximal insertion of M.m. musculus, and the two linked insertions of M.m. musculus are separated by an inverted segment of euchromatin. Taking into account all these facts, it is easy to suppose that the aberrant chromosome 1 of $M . m$. musculus has arisen from an aberrant chromosome, which was very similar to the aberrant chromosome 1 existing in recent populations of M.m. domesticus, as a result of single inversion. The proximal breakpoint occurred inside the HSR and the distal one between sub-bands E3 and E4. From this point of view, the hypothesis of an independent origin of HSRs in M.m. musculus and M.m. domesticus might be rejected. Furthermore, these data can be interpreted as evidence of a monophiletic origin of the aberrant chromosome 1, which is now 
spreading in feral populations of house mouse from the Volga to the Far East.

One could argue that the simple inversion hypothesis proposed above, is inadequate to explain the origin of the double insertion, from the single insertion, because it does not account for the duplication. Two steps seem to be required: a tandem duplication of the HSR, followed by an inversion of one HSR together with distal bands $\mathrm{D}$ and $\mathrm{E}$. This argument would be valid for normal chromosomal material. However we are dealing with HSR, which represents amplified sequences of DNA, and a wide variation in size has been described in both subspecies. It is therefore now impossible to reconstruct the evolutionary changes that have occured in the size of such HSRs. The inversion could have arisen in a chromosome carrying a very long (extensively amplified) HSR, or increase in size of the insertions, could have taken place after inversion, as a result of new amplification events. Taking into account the highly repetitive nature of the HSR one can envisage the loss of part of the progenitor HSR as the cause of the smaller average size of the $M . m$. domesticus HSR, in comparison to the HSR found in M.m. musculus.

From the results of the analyses of mitochondrial DNA patterns (Ferris et al., 1983), and of the isozyme spectrum (Thaler et al., 1981), the time of divergence of the subspecies M.m. musculus and M.m. domesticus has been estimated as being 1-1.5 millions years ago. These data suggest that the chromosomes carrying the HSRs have been maintained in natural populations of house mouse over a long time. Their frequencies in Siberian as well as in European populations are rather high. However the causes of their widespread occu rrence and adaptive significance are still unclear.

\section{REFERENCES}

AGULNIK, S. I., GORLOV, I. P. AND AGULNIK, A. I. 1988. New variant of chromosome 1 of house mouse. Citologija, 30 , 773-775. (In Russian).

FERRISS, D., SAGE, R. D., PRACER, E. M., RITTE, U. AND WILSON, A. C. 1983. Mitochondrial DNA evolution in mice. Genetics, 105, 681-721.

IKEUCHI, T. 1984. Inhibitory effect of ethidium bromide on mitotic chromosome condensation and its application to high resolution chromosome banding. Cytogenet. Cell Genet., 38, 56-61.

LYON, M. F. 1981. Rules for nomenclature of chromosome anomalies. In Green, M. C. (ed.), Genetic Variants and Strains of the Laboratory Mouse, Gustav Fisher Verlag, Stuttgart-N.Y., pp. 314-315.

THALER, L., BONHOMME, F. AND BRITTON-DAVIDIAN, J. 1981. Processes of speciation and semi-speciation in the house mouse. In Berry, R. J. (ed.), Biology of the House Mouse, Academic Press, N.Y., pp. 27-41.

TRAUT, W., WINKING, H. AND ADOLPH, s. 1984. An extra segment in chromosome 1 of wild Mus musculus: C-band positive homogeneously staining region. Cytogenet. Cell Genet., 38, 290-297.

WEITH, A., WINKING, H., BRACKMANN, W., BOLDYREFF, W. AND TRAUT, w. 1987. Microclones from a mouse germ line HSR detect amplification and complex rearrangements of DNA sequences. EMBO J., 6, 1295-1299.

YAKIMENKO, L. W. AND KOROBITSINA, K. V. 1988. Rare variant of chromosome 1 of the house mouse: arising from two additional heterochromatic segments. Genetika, 24, 376-378. (In Russian). 